\title{
Beam forming and modelling in cognitive radio wireless networks
}

\author{
${ }^{1}$ Mohammad Javad Davoudpoor, 2Rouhollah Aghajani \\ IM.A student, Telecommunications engineering, Islamic Azad University of Najafabad \\ Assistant professor, Islamic Azad University of Najafabad2
}

\begin{abstract}
This study is aimed at using modern methods in beam forming in cognitive radio networks. The beam shaper has an important role in wireless communication by interferences reduction and system capacity improvement. Utilizing this method, the signal of every user is sent as much as feasible by base stations toward the receiver and interferences in the system are effectively reduced. It is worth mentioning that for implementing this method, it is needed to have enough information for forming sender antennas in the best way in order to reach the minimum interference and maximum cognitive radio link efficiency. This information is received in the sender in various ways. The goal of this survey is statingthe pattern forming methods and presenting modern approaches based on information simplification for performing this process while relatively maintaining the system quality. According to an adaptive structure in this study, whole of the channel information is simply sent from the receiver to the sender and it is not necessary to send all the channel information in the time of the channel change. Using this information in forming the pattern by the sender, the system quality would remain satisfactory and desirable. In another approach, quasi-optimal propagation pattern shaping vector is used by the purpose of simplification, convergence of the equations presented, and the elimination of limitations emerged.
\end{abstract}

Keywords: Cognitive radio, beam forming, adaptive structure, interference

\section{Introduction}

Designing and implementing wireless links with high speed and quality is a challenge in telecommunication researches. The popularity of cellular, personal communication, and other emerging telecommunication systems are of the factors to the need for a new generation of telecommunication networks. Rapid growth in demand for cellular telecommunications serviceshas been depicted in figure 1-1.

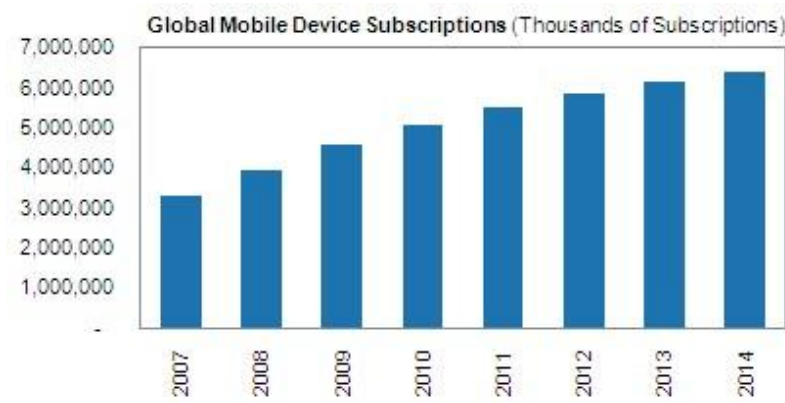

Figure 1-1: Rapid growth in demand for cellular telecommunications services [1]

In the previous decade, eye-catching development of telecommunication technology and the tendency increase in using wireless devices and subsequently the demand for high rates have significantly heightened the need for having an electromagnetic spectrum. Due to the way of designation and use of the current spectrum, the available spectrum resources cannot meet this growing need. This work leads to very low performance in some of the frequencies and working congestion at other frequencies specially the general ones. As demonstrated in figure (1-2), a considerable section of the spectrum is remained useless. 


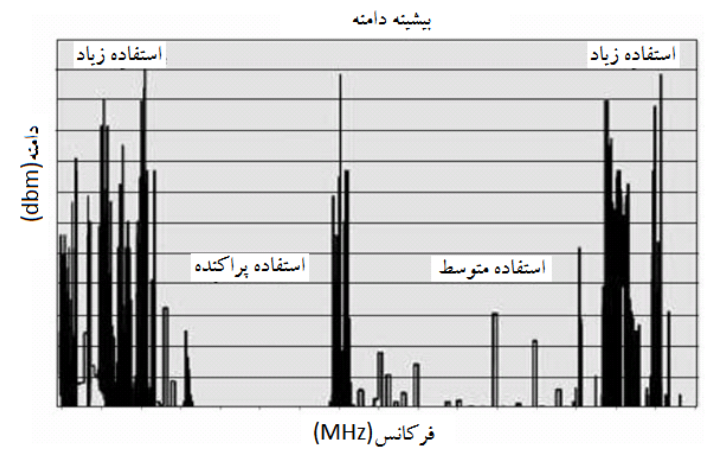

Figure 1-2: The use of different bands in the frequency spectrum [2]

Cognitive radio is a concept for wireless communication which according to that, a network or a wireless node changes its send and receive parameters so as to provide the service quality for its users and simultaneously reduce the interference with valid users. The cognitive radio users called secondary users have the capability of using the primary users' spectrum in a specific place or time while primary users are licensed to utilize the spectrum absolutely. Cognitive radio is capable of using the temporary free spectrum called white space or spectrum hole. In case this band is occupied with a valid user, the cognitive radio goes to another free spectrum or stays in the same band. However, it leads to some changes to reduce interferences in the sending power, modulation or propagation pattern. As shown in figure (1-3), cognitive radio uses frequency gaps through dynamic access.

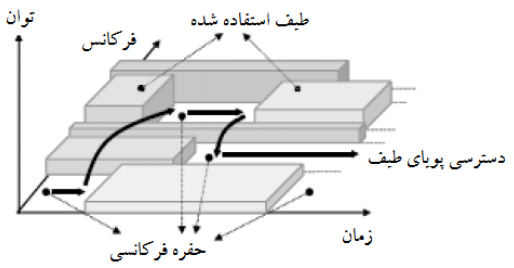

Figure 1-3: The concept of frequency gap [2]

Some of the cognitive radio basic technologies are currently used in a small number of mobile networks including cellular networks, wireless telephones, and local mobile networks. Executing the cognitive cycle requires flexibility in the cognitive systems configuration. Such capability and adjustability of performance parameters guarantees the hardware components without modification and makes the cognitive radio to be easily adapted to the dynamic environment. Performance frequency, modulation, sending power, and telecommunication technology are of the configuration parameters registered so far. It should be emphasized that the cognitive radio sending parameters are capable of being configured not only in the beginning of information transfer but also during the transition. These factors are changed according to the spectrum so as the cognitive radio can go to another band. Therefore, it is needed for new send and receive parameters to be formed and telecommunication and modulation parameters to be used. The antennas beam formation is done with the purpose of no interference in the primary and secondary users spectrum. The propagation pattern formation needs some information in order to optimally determine the antennas weight coefficients. The aim of this study is to propose a simple method in presenting the information required for improving the antennas propagation pattern which reduces cost and computation loads while maintaining the quality at a satisfactory and desirable level.

Propagation pattern formation

Generally, the propagation pattern formation can be defined as the process of angular separation for an antenna. 


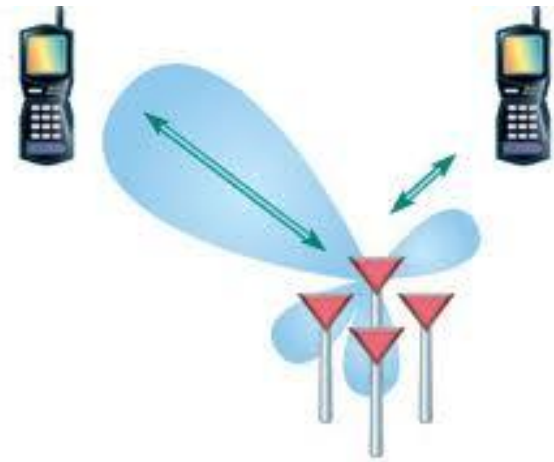

Figure 1-4: The propagation pattern formation in the cognitive radio network

The propagation pattern former contains an array of antennas which each of them is placed in a special position. The output of each antenna is appropriately filtered. In addition, other outputs caused by the other antennas and added to the optimal antenna output are also filtered.Some of the merits to the use of propagation pattern formers are the group delay reduction, multipath feeding, interference reduction, capacity and spectrum yield improvement, and transfer efficiency increase.Forming the propagation pattern in cognitive radio is generally divided to the twofollowing categories.

The receiver distribution pattern former (Upward): These types of propagation pattern formers are a classic technique in numerous applications including radars, sonars, telecommunications, astronomy, and seismology. This tactic increases Signal-to-Interference-Plus-Noise Ratio (SINR) and reduces the co-channel interference and Inter-Symbol Interference (ISI). Figure (1-5) demonstrates an example of the propagation pattern former in the receiver.

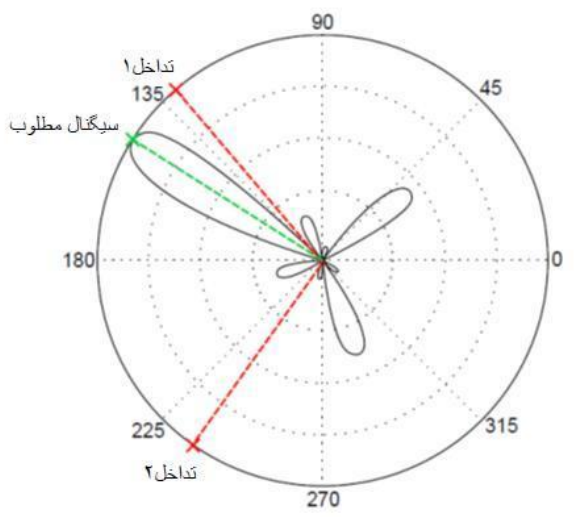

Figure: 1-5: An instance of the propagation pattern former in the receiver

In case the information of optimal signal vector is concomitant with error in conventional techniques, the performance is significantly decreased [4] and [9-11]. This performance directs the design toward optimization in the worst situation [12-19]. The distribution pattern formers vector is obtained while assuming that the limitations output possibility is lower than what has been determined.Convex optimization is used for solving the problems in these methods.

Distribution pattern former of the transmitter (Downward): The coefficients vector is designed similar to the receiver propagation pattern former. However, the multiuser distribution pattern former vector is designed for various users. These vectors are furnished with the purpose of interference reduction and quality of service improvement [5 and 6] and [25]. The upward propagation pattern former uses an array of antennas for a specific user and does not affect the other users signal in addition to be designed independent of any receiver [5 and 6] and [26 and 27]. The sender and receiver propagation pattern former vectors also have differences in accessing the channel state information. Depicted in figure (1-6) is an example of downward propagation pattern which uses multiple antennas for transferring the information toward the two users. 


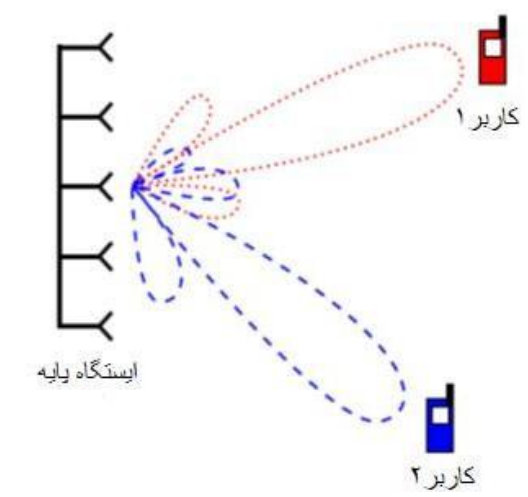

Figure 1-6: An instance of the propagation pattern former in the sender

There are some techniques used for designing the sender distribution pattern formers which would be ideal if the channel information is known [5 and 6] and [32 and 34]. However, it is impossible at work and the channel is not ideally accessible in the receiver.

\section{Channel estimation}

The channel changes the signal in various ways such as small and large scale path losses. The path loss factors in large scale are diffraction, reflection, and scattering. Moreover, the signal is weakened in the propagation path from the sender to the receiver. Different algorithms have been presented for channel estimation and data retrieving. The channel estimation approaches are briefly stated as the followings.

\section{Training -Based Estimation}

In this method, the training sequences are sent along the channel symbols transition. Number of sequences in noise-less channel is equal to the channel impulse response length. Furthermore, due to heightening the estimation accuracy, this number is increased when the channel has noise.

\section{Blind estimation}

Blind algorithms work based on the signal general properties for recovering the channel. For instance, assuming that modulation type, carrier frequency, signal coding type and so forth are known, the unknown channel can be estimated.

\section{Semi-blind estimation}

This is a mixture of the two previous estimations which approximates the channel using the training sequences and other signal limitations.

\section{Data-aided channel estimation}

The main or maybe the most important reason to the use of channel estimation is to utilize estimation output for data retrieving. The data revealed can be used for channel estimation improvement. Furthermore, repetitive techniques can be utilized to enhance channel and data recovery. The designer can implement the above methods using batch processing or adaptive algorithms. There are numerous advantages to estimation by adaptive or duplicated methods. Its best merit is that although the estimator direct implementation may be complicated, the designer can execute the same algorithm adaptively or repetitively.

\section{Simulation findings}

According to the model supposed, the simulations are done by sixprimary users, antenna $\mathrm{M}=7, \mathrm{P}_{\mathrm{M}}=$ 2.5 , and interference threshold of $10 \mathrm{~dB}$. In addition, the secondary user channel has been considered as a complex Gaussian vector with the average of 0 and variance of 1 . In order to reach logical results in simulation process, it is assumed that the primary users have been located around the secondary users with Gaussian channel vector in radius of $\mathrm{R}$. The amount of interference, secondary link efficiency, and consumption power have been drawn in the proposed method based on procedure mentioned in [46]. The findings obtained for different values of $R$ through execution of the two algorithms have been illustrated in figures (4-2) and (4-5). 


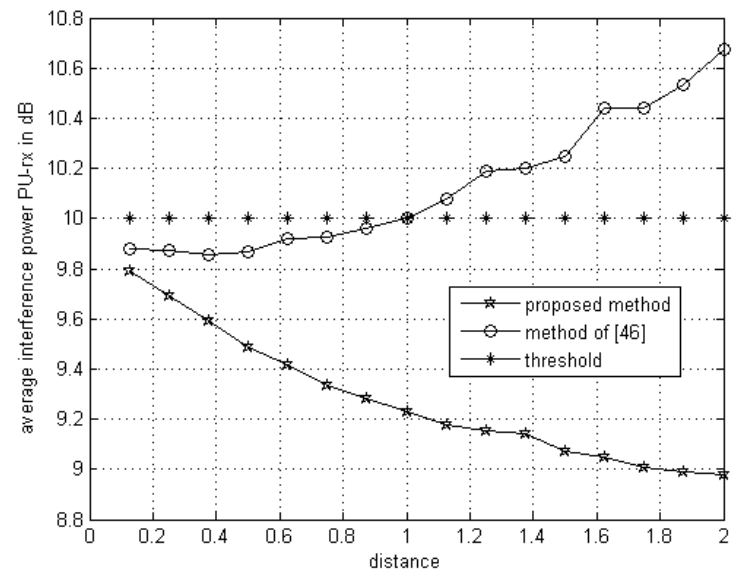

Figure 2-1: Interface average based on radius increase between the primary and secondary users.

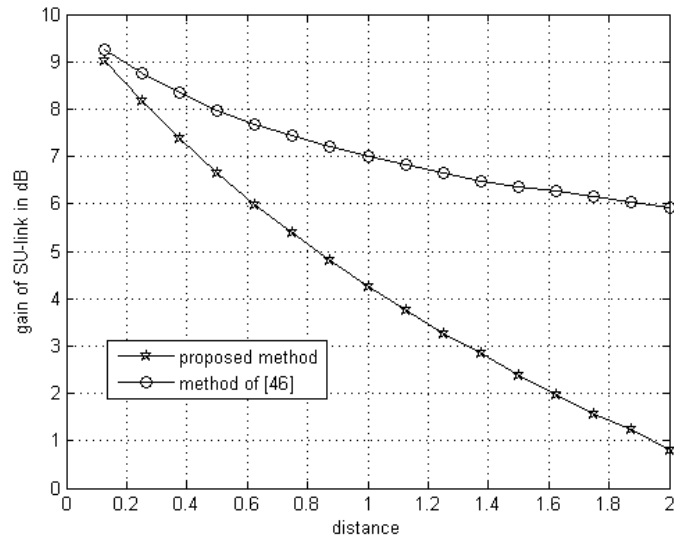

Figure 2-2: Secondary user link efficiency according to radius increase between the primary and secondary users

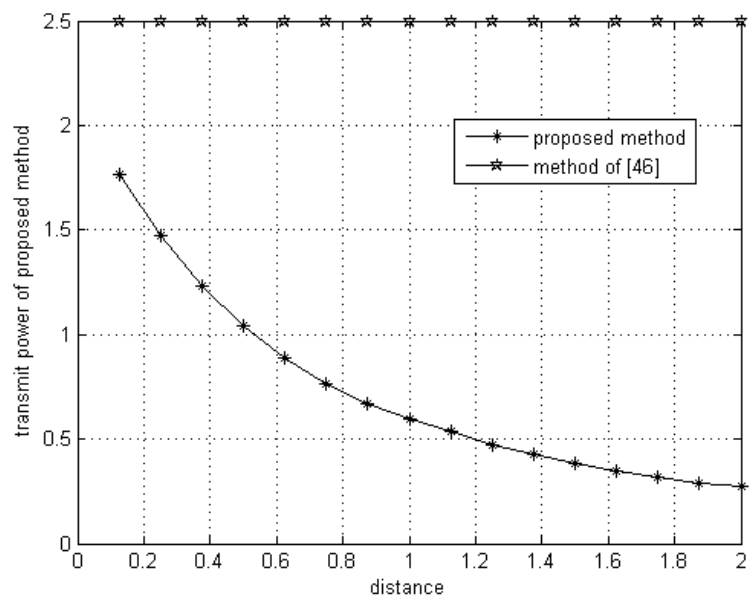

Figure 2-3: consumption power based on the radius increase between initial and secondary users

The method used in [36] does not maintain the interference while the proposed method is simple and keeps the interference threshold. Moreover, the consumption power is very low in the method proposed, however; the efficiency is less than the method used in [46]. Number of users can be increased in order to reach the following results. In figures below, numbers of theprimary users and secondary sender antennas are $\mathrm{m}=10$ and $\mathrm{M}=11$, respectively. 


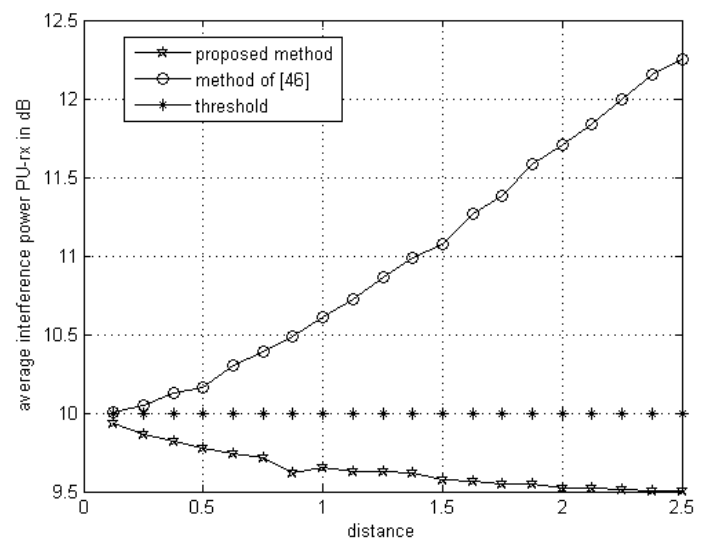

Figure 2-4: Interference average based on radius increase between the primary and secondary users

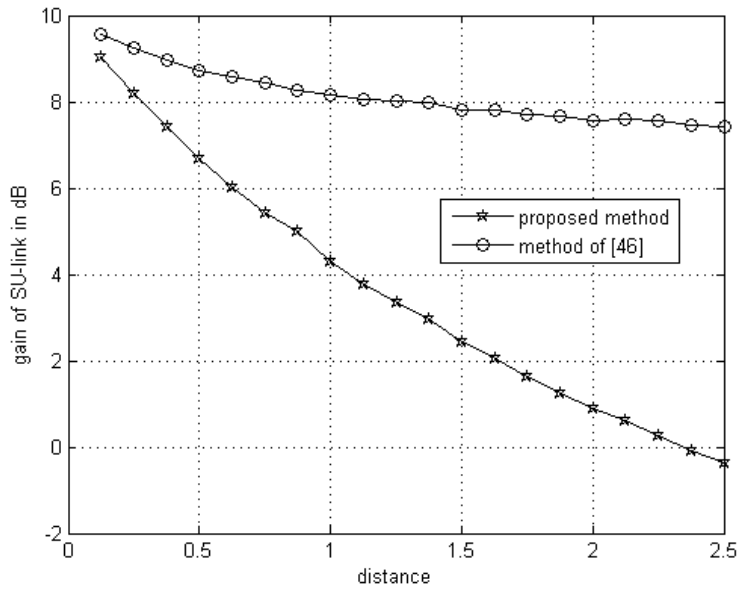

Figure 2-5: The secondary user link efficiency according to radius increase between the primary and secondary users.

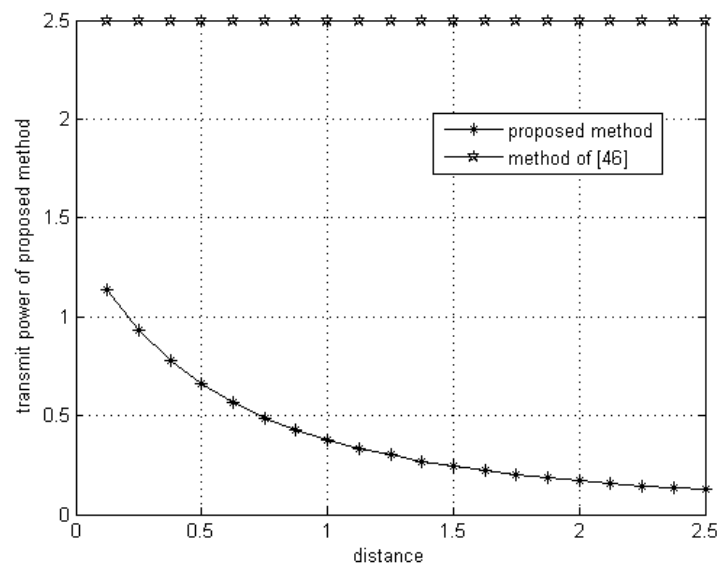

Figure 2-6: Power consumption based on radius increase between the primary and secondary users

Two secondary users and threeprimary users share their spectrum. Complex Gaussian vector of variable channels has the average of 0 and variance of 1 . CVX optimization software has been used for the proposed method simulation and repetitive algorithm of [42] has also been executed. The consumption power in these two methods have been compared with changes in the secondary channels variance. 


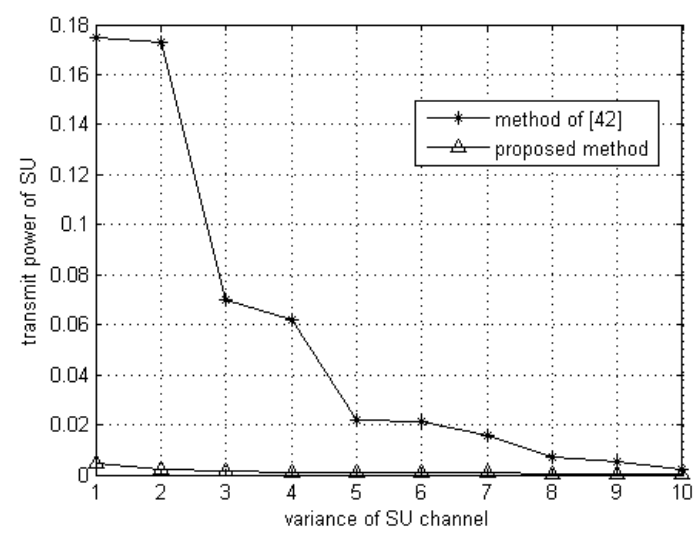

Figure 2-7: Optimal power in the method and the proposed method with assumption of the secondary channel changes.

\section{Conclusion}

Cognitive radio technology is a technique for increasing spectrum efficiency. Distribution pattern forming is aimed at preventing cognitive radio users' interference with primary users and improving secondary users' spectrum utilization. Channel estimation methods were examined first. Then, two approaches were presented in the field of propagation pattern formation. The model of the first method was used in the proposed method and concise information was sent from the primary and secondary receivers to the secondary sender by the help of partial feedback. It was seen that these brief data were so helpful for maintaining interference in the ranged considered. As revealed in simulation results, interference cannot be kept in the determined range as the feedback is disconnected. Furthermore, using the channel fault function analysis, channel vectors sending from primary and secondary receivers were done so as the feedback bits reach the minimum. Cognitive radio user can adaptively adjust his distribution pattern former vector. Pattern formation can be done by considering two states of ZF and MRT (Optimal propagation pattern former). In the moments in which higher efficiency is needed, MRT technique is utilized and in cases that interference limitation is considered to be zero, ZF propagation pattern former is used.

\section{References}

[1]. $\quad$ www.isuppli.com/mobile-and -wireless-communications.

[2]. I.F. Akyildiz, W.Y. Lee, M.C. Vuran, S.Mohanty "NeXt generation/dynamic spectrum access/cognitive radio wireless networks: A survey" ELSEVIER Computer Networks 50,p2127-2159, May 2006.

[3]. Bhimo Wikan Hantoro " Distributed Beamforming for Cognitive Radio Networks", MSc Thesis,Delft University of Technology, August 2011.

[4]. A. B. Gershman, N. D. Sidiropoulos, S. Shahbazpanahi, M. Bengtsson, and B. Ottersten, “Convex optimization-based beamforming: From receive to transmit and network designs," IEEE Signal Processing Magazine, vol. 27, no. 3, pp. 62-75, May 2010.

[5]. F. R. Farrokhi, L. Tassiulas, and K. J. R. Liu, "Joint optimal power control and beamforming in wireless networks using antenna arrays," IEEE Transactions on Communications, vol. 46, no. 10, pp. 1313-1324, Cct. 1998.

[6]. F. R. Farrokhi, K. J. R. Liu, and L. Tassiulas, "Transmit beamforming and power control for cellular wireless systems," IEEE Journal on Selected Areas in Communications, vol. 16, no. 8, pp. 1437-1450, Oct 1998.

[7]. T. W. Monzingo, R. A. andMiller, Introduction to Adaptive Arrays, SciTech Publishing,New York, 1980.

[8]. H. L. Van Trees, Optimum Array Processing, Wiley, New York, 2002.

[9]. H. Cox, R. Zeskind, and M. Owen, "Robust adaptive beamforming," IEEE Transactions on Acoustics, Speech and Signal Processing, vol. 35, no. 10, pp. 1365-1376, Oct. 1987.

[10]. H. Cox and R. Pitre, "Robust DMR and multi-rate adaptive beamforming," in Conference Record of the Thirty-First Asilomar Conference on Signals, Systems \& Computers, 1997, Nov. 1997, vol. 1, pp. 920-924.

[11]. A. B. Gershman, "Robust adaptive beamforming in sensor arrays," AEU International Journal of Electronics and Communications, vol. 53, pp. 305-314, Dec.1999.

[12]. S. Shahbazpanahi, A. B. Gershman, Z.-Q. Luo, and K. M. Wong, "Robust adaptive beamforming for general-rank signal models using worst-case performance optimization,"in Sensor Array and Multichannel Signal Processing Workshop Proceedings,2002, Aug. 2002, pp. 13-17.

[13]. S. A. Vorobyov, A. B. Gershman, and Z.-Q. Luo, "Robust adaptive beamforming using worst-case performance optimization: a solution to the signal mismatch problem,"'IEEE Transactions on Signal Processing, vol. 51, no. 2, pp. 313-324, Feb. 2003.

[14]. S. Shahbazpanahi, A. B. Gershman, Z.-Q. Luo, and K. M. Wong, "Robust adaptive beamforming for general-rank signal models," IEEE Transactions on Signal Processing, vol. 51, no. 9, pp. 2257-2269, Sep. 2003.

[15]. S. A. Vorobyov, A. B. Gershman, Z.-Q. Luo, and N. Ma, "Adaptive beamforming with joint robustness against mismatched signal steering vector and interference nonstationarity," IEEE Signal Processing Letters, vol. 11, no. 2, pp. 108-111, Feb. 2004.

[16]. R. G. Lorenz and S. P. Boyd, "Robust minimum variance beamforming," IEEE Transactions on Signal Processing, vol. 53, no. 5, pp. 1684-1696, May 2005.

[17]. A. El-Keyi, T. Kirubarajan, and A. B. Gershman, "Wideband robust beamforming based on worst-case performance optimization," in IEEE SSP Workshop, Bordeaux, France, July 2005, pp. 265-270. 
[18]. S.-J. Kim, A. Magnani, A. Mutapcic, S. P. Boyd, and Z.-Q. Luo, "Robust beamforming via worst-case SINR maximization," IEEE Transactions on Signal Processing, vol. 56, no. 4, pp. 1539-1547, Apr. 2008.

[19]. M. Rubsamen and A. B. Gershman, "Robust presteered broadband beamformingbased on worst-case performance optimization," in 5th IEEE Sensor Array and Multichannel Signal Processing Workshop, SAM'08., July 2008, pp. 340-344.

[20]. Z.-Q. Luo and W. Yu, "An introduction to convex optimization for communicationsand signal processing," IEEE Journal on Selected Areas in Communications, vol. 24, no. 8, pp. 1426-1438, Aug. 2006.

[21]. Y. Rong, S. A. Vorobyov, and A. B. Gershman, "A robust linear receiver for uplink multi-user MIMO systems based on probability-constrained optimization and secondorder cone programming," in IEEE Sensor Array and Multichannel Signal Processing Workshop Proceedings, 2004, July 2004, pp. 153-157.

[22]. Y. Rong, S. A. Vorobyov, and A.B. Gershman, "Robust linear receiver design for multi-access space-time block coded MIMO systems using stochastic optimization," in IEEE Workshop Statistical Signal Processing, Bordeaux, France, Jul. 2005 , pp. 65-70.

[23]. S. A. Vorobyov, Y. Rong, and A. B. Gershman, "Robust minimum variance adaptive beamformers and multiuser MIMO receivers: From the worst-case to probabilistically constrained designs," in IEEE International Conference on Acoustics, Speech and Signal Processing, ICASSP'06, May 2006, vol. 5.

[24]. S. A. Vorobyov, H. Chen, and A. B. Gershman, "On the relationship between robust minimum variance beamformers with probabilistic and worst-case distortionless response constraints," IEEE Transactions on Signal Processing, vol. 56, no. 11, pp. 57195724, Nov. 2008.

[25]. C. Farsakh and J. A. Nossek, "Spatial covariance based downlink beamforming in an SDMA mobile radio system," IEEE Transactions on Communications, vol. 46, no. 11, pp. 1497-1506, Nov. 1998.

[26]. L. C. Godara, "Applications of antenna arrays to mobile communications. I. Performance improvement, feasibility, and system considerations," Proceedings of the IEEE, vol. 85, no. 7, pp. 1031-1060, July 1997.

[27]. L. C. Godara, "Application of antenna arrays to mobile communications. Beamforming and direction-of-arrival considerations," Proceedings of the IEEE, vol. 85,no. 8, pp. 1195-1245, Aug. 1997.

[28]. Kay S. M., Fundamentals of Statistical Signal Processing: Detection Theory, vol. 2, Prentice-Hall, Englewood Cliffs, NJ, 1998.

[29]. M. Bengtsson and B. Ottersten, "Optimal and suboptimal transmit beamforming," in Handbook of Antennas in Wireless Communications, L. C. Godara, Ed., chapter 18. CRC Press, Aug. 2001.

[30]. D. Gerlach and A. Paulraj, “Adaptive transmitting antenna arrays with feedback,"IEEE Signal Processing Letters, vol. 1, no. 10, pp. 150-152, Oct. 1994

[31]. D. Samardzija and N. Mandayam, "Unquantized and uncoded channel state information feedback in multiple-antenna multiuser systems," IEEE Transactions on Communications, vol. 54, no. 7, pp. 1335-1345, July 2006

[32]. E. Visotsky and U. Madhow, "Optimum beamforming using transmit antenna arrays,"in IEEE 49th Vehicular Technology Conference, 1999, Jul 1999, vol. 1, pp. 851-856.

[33]. E. Karipidis, N.D. Sidiropoulos, and Zhi-Quan Luo, "Quality of service and maxmin fair transmit beamforming to multiple cochannel multicast groups," IEEE Transactions on Signal Processing, vol. 56, no. 3, pp. 1268-1279, March 2008

[34]. N. Bornhorst and M. Pesavento, "An iterative convex approximation approach for transmit beamforming in multi-group multicasting," in IEEE 12th International Workshop on Signal Processing Advances in Wireless Communications, SPAWC'11, June 2011, pp. 426-430.

[35]. B. Hassibi and B. M. Hochwald, "How much training is needed in multiple-antennawireless links," IEEE Transactions on Information Theory, vol. 49, no. 4, pp. 951-963, April 2003

[36]. D. J. Love, R. W. Heath, W. Santipach, and M. L. Honig, "What is the value oflimited feedback for MIMO channels?," IEEE Communications Magazine, vol. 42,no. 10, pp. 54-59, oct. 2004.

[37]. B. K. Chalise and A. Czylwik, "Robust uplink beamforming based upon minimumoutage probability criterion," in IEEE Global Telecommunications Conference,GLOBECOM '04, Dec. 2004, vol. 6, pp. 3974-3978

[38]. B. K. Chalise, S. Shahbazpanahi, A. Czylwik, and A. B. Gershman, "Robust downlink beamforming based on outage probability specifications," IEEE Transactions on Wireless Communications, vol. 6, no. 10, pp. 3498-3503, October 2007.

[39]. Pesavento, M.; Ciochina, D.; Gershman, A.B., "Iterative dual downlink beamforming forcognitive radio networks," Cognitive Radio Oriented Wireless Networks \& Communications(CROWNCOM), 2010 Proceedings of the Fifth InternationalConference on , vol., no., pp.1,5, 9-11 June 2010.

[40]. Lan Zhang; Ying-Chang Liang;Yan Xin;H. Vincent Poor, "Robust cognitive beamforming with partial channel state informatio," Wireless Communications, IEEE Transactions on , vol.8,no.8, August 2010.

[41]. K. Huang and R. Zhang, "Cooperative feedback for multiantenna cognitive radio networks," IEEE Trans. Signal Process., vol. 59, pp. 747-758,Feb. 2011.

[42]. Bakr, O.; Johnson, M.; Mudumbai, R.; Ramchandran, K., "Multi-Antenna Interference Cancellation Techniques for Cognitive Radio Applications," Wireless Communications and Networking Conference, 2009. WCNC 2009. IEEE , vol., no., pp.1,6, 5-8 April 2009.

[43]. Gharavol, E.A.; Ying-Chang Liang; Mouthaan, K., "Robust Downlink Beamforming in Multiuser MISO Cognitive Radio Networks With Imperfect Channel-State Information,"VehicularTechnology, IEEE Transactions on, vol.59, no.6, pp.2852,2860,July 2010.

[44]. Huang, Yongwei; Qiang Li; Wing-Kin Ma; Zhang, Shuzhong,"Robust Multicast Beamforming for SpectrumSharingBasedCognitiveRadios,"SignalProcessing,IEEETransaction on,vol.60,no.1,pp.527,533,Jan.2012.

[45]. Wajid, I.; Pesavento, M.; Eldar, Y.C.; Ciochina, D., "RobustDownlink Beamforming With Partial Channel State Informationfor Conventional and Cognitive Radio Networks," Signal Processing, IEEE Transactions on , vol.61, no.14, pp.3656,3670,July15, 2013.

[46]. Jung-Hoon Noh; Seong-Jun Oh, "Beamforming in a Multi-UserCognitive Radio System with Partial Channel State Information,"Wireless Communications, IEEETransactions on , vol.12, no.2, pp.616,625,February2013.

[47]. U. Madhow, K. Bruvold, and L. J. Zhu, "Differential MMSE: Aframework for robust adaptive interference suppression for DSCDMAover fading channels," IEEE Transactions on Communications, vol. 53, no. 8, pp. 1377-1390, Aug. 2005.

[48]. Kamal Shahtalebi,Gholamreza Bakhshi, "A simple adaptive partial feedback method for full multiple input multiple output channel estimation", IET Communications,August 2012. 